\title{
ROLE OF ARSENIC HEXAGONAL GROWTH-SUPPRESSION ON A CUBIC GaNAs GROWTH USING METALORGANIC CHEMICAL VAPOR DEPOSITION
}

\author{
S. Yoshida*, T. Kimura**, J. Wu**, J. Kikawa*, K. Onabe**, and Y. Shiraki*** \\ *Yokohama R\&D Laboratories, The Furukawa Electric Co., Ltd. \\ 2-4-3 Okano, Nishi-ku, Yokohama 220-0073, Japan \\ **Department of Applied Physics, The University of Tokyo, \\ 7-3-1 Hongo, Bunkyo-ku, Tokyo 113-8654, Japan \\ ***Research Center for Advanced Science and Technology, The University of Tokyo, \\ 4-6-1 Komaba, Meguro-ku, Tokyo 153-8904, Japan
}

\begin{abstract}
The hexagonal domain suppression-effects in cubic-GaNAs grown by metalorganic chemical-vapor deposition (MOCVD) is reported. A thin buffer layer $(20 \mathrm{~nm})$ was first grown on a substrate at $853 \mathrm{~K}$ using trimethylgallium and dimethylhydrazine (DMHy), and GaNAs samples were grown at different $\mathrm{AsH}_{3}$ flow rates $(0 \sim 450 \mu \mathrm{mol} / \mathrm{min})$ at $1193 \mathrm{~K}$. As a result, three types of surface morphologies were obtained: the first was a smooth surface $\left(\mathrm{AsH}_{3}=0 \mu \mathrm{mol} / \mathrm{min}\right)$; the second was a mirrorlike surface having small and isotropic grains $\left(\mathrm{AsH}_{3}: 45 \sim 225 \mu \mathrm{mol} / \mathrm{min}\right)$; and the third involved threedimensional surface morphologies (above $450 \mu \mathrm{mol} / \mathrm{min}$ of $\mathrm{AsH}_{3}$ flow rate). Furthermore, it was confirmed using X-ray diffraction that the mixing ratio of hexagonal GaNAs in cubic GaNAs decreased with an increase of the $\mathrm{AsH}_{3}$ flow rate. We could obtain GaNAs having a cubic component of above $85 \%$ at $\mathrm{AsH}_{3}$ flow rates above 20 $\mu \mathrm{mol} / \mathrm{min}$. Therefore, the MOCVD growth method using $\mathrm{AsH}_{3}$ and $\mathrm{DMHy}$ was mostly effective for suppressing hexagonal GaNAs. It was observed that the photoluminescence intensity of GaNAs was decreased with increase of arsine flow rate.
\end{abstract}

\section{INTRODUCTION}

III-N-V materials, such as GaNP and GaNAs, have a very large band-gap bowing and are very promising for light-emitting devices with a very wide wavelength region from ultraviolet to infrared [1,2]. They are expected to realize a wider range of bandgaps with a smaller lattice mismatch to GaN.

However, III-N-V type alloys can be hardly grown under an equilibrium condition, since they have a very large miscibility gap $[3,4]$. The gas-source molecular-beam epitaxy (GSMBE) growth of nitrogen $(\mathrm{N})$ rich hexagonal (h) GaNAs [5] and GaNP [6,7] alloys on sapphire substrates and their optical properties have been recently reported. The maximum As and $\mathrm{P}$ concentrations of these alloys with a hexagonal structure are so far limited to $0.26 \%$ [6] and $8.3 \%$ [7], respectively. However, there has been no report on a metalorganic chemical vapor deposition (MOCVD) growth of cubic-GaNAs using 
the dimethylhydrazine (DMHy). In this paper, the As effect for hexagonal growthsuppression on cubic GaNAs growth is reported in MOCVD.

\section{EXPERIMENTAL}

GaNAs was grown on a GaAs (001) substrate using a horizontal MOCVD apparatus. The pressure in the reactor was 160 Torr. Trimethylgallium (TMG), DMHy $\left[\left(\mathrm{CH}_{3}\right)_{2} \mathrm{NNH}_{2}\right]$ and arsine $\left(\mathrm{AsH}_{3}\right)$ were used as precursors of $\mathrm{Ga}, \mathrm{N}$, and As, respectively. A GaN buffer layer of about $20 \mathrm{~nm}$ in thickness was grown on the substrate using TMG and DMHy at $853 \mathrm{~K}$. After that, a GaNAs layer was grown on the $\mathrm{GaN}$ buffer layer at $1193 \mathrm{~K}$ under the conditions of different $\mathrm{AsH}_{3}$ flows. The growth temperature was constant at $1193 \mathrm{~K}$, since when the growth temperatures were lower than $1193 \mathrm{~K}$ and also higher than $1223 \mathrm{~K}$, the grown surface became rough. The surface morphologies of the samples were observed by scanning electron microscopy (SEM), and the surface roughness was measured by atomic force microscopy (AFM). The crystal structure was investigated by an X-ray diffraction (XRD) measurement. Secondary ion mass spectrometry (SIMS) was carried out to estimate As incorporation into the c-GaN films. To investigate the optical properties, a photoluminescence measurement of GaNAs was carried out using a He-Cd laser $(325 \mathrm{~nm})$ at $5 \mathrm{~K}$.

\section{RESULTS AND DISCUSSION}

In order to increase the As incorporation into cubic GaNAs, the $\mathrm{AsH}_{3}$ flow rate was changed from 0 to $450 \mu \mathrm{mol} / \mathrm{min}$ and the flow rates of TMG and DMHy were fixed at 18 and $230 \mu \mathrm{mol} / \mathrm{min}$, respectively. Without $\mathrm{AsH}_{3}$ flow, the growth rate of $\mathrm{c}-\mathrm{GaN}$ was $1.5 \mathrm{~nm} / \mathrm{sec}$. When the $\mathrm{AsH}_{3}$ flow was increased to $450 \mu \mathrm{mol} / \mathrm{min}$, the growth rate was reduced to $1.0 \mathrm{~nm} / \mathrm{sec}$. The thickness of the epitaxial layers was approximately $600 \mathrm{~nm}$. Figure 1 shows the surface morphologies of the samples at different $\mathrm{AsH}_{3}$ flow rates ranging from 0 to $450 \mu \mathrm{mol} / \mathrm{min}$, observed by SEM. Figure 1 (a) shows GaN surface morphologies without $\mathrm{AsH}_{3}$ flow. The shape of the grains is elongated in the [110] direction and the length of each grain is several microns. These grains have a tendency to form (111) B facets and (113) A facets [8]. Figure 1 (b) shows the surface morphologies in the case of $\mathrm{AsH}_{3}$ flow under $225 \mu \mathrm{mol} / \mathrm{min}$. The surface was smooth and the grains were smaller with more isotropic shapes. When the $\mathrm{AsH}_{3}$ flow rate was, moreover, as large as $450 \mu \mathrm{mol} / \mathrm{min}$, the GaNAs growth was more three dimensional and the grains were also isotropic and larger.

We also observed the AFM images in order to investigate the surface roughness of GaNAs layers grown at different $\mathrm{AsH}_{3}$ flow rates $(0 \sim 225 \mu \mathrm{mol} / \mathrm{min})$. It was found that the surface became smoother with increasing the $\mathrm{AsH}_{3}$ flow rate. That is, the rootmean-square (rms) showing a surface roughness became smaller than $22.7 \mathrm{~nm}$ with increasing the $\mathrm{AsH}_{3}$ flow rate. The above-mentioned results of SEM and AFM indicate that with $\mathrm{AsH}_{3}$ the grain size and shape was smaller and more isotropic, and that the surface was smoother, although the growth rate decreased wth increasing $\mathrm{AsH}_{3}$ flow. 
This is considered to be due to As adsorption on the GaN surface during the growth process. It is considered that As adsorption on the GaN surface lowers the surface energy to stabilize the (001) surface, resulting in suppressing the generation of a hexagonal structure, as Foxon et al previously reported [9].

Figure 2 shows three-dimensional maps of XRD of the two grown layers with and without $\mathrm{AsH}_{3}$ flow. The X-ray incident azimuth was the $\{1-10\}$ direction. Two different diffraction peaks from h-GaN (1-101) were observed along with the main diffraction from c-GaN (002), as shown in Figure 2 (a). With this X-ray incident azimuth, the hGaN (1-101) domains, holding on two different (111) B surfaces, were observed. The
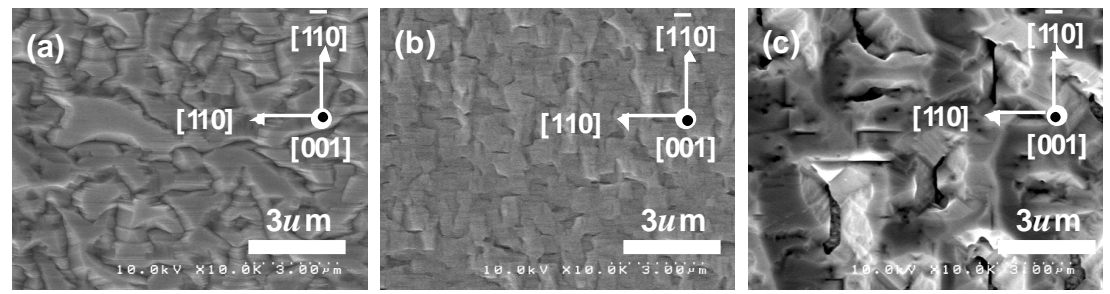

Figure 1. SEM images of samples with various $\mathrm{AsH}_{3}$ flow rates ranging from 0 to 450 $\mu \mathrm{mol} / \mathrm{min}$. (a) $\mathrm{AsH}_{3}=0 \mu \mathrm{mol} / \mathrm{min}, \quad \mathbb{I}=\mathrm{AsH}_{3} /\left(\mathrm{AsH}_{3}+\mathrm{DMHy}\right)=0, \quad$ (b) $\mathrm{AsH}_{3}=225$ $\mu \mathrm{mol} / \mathrm{min}$, Il=0.49, (c) $\mathrm{AsH}_{3}=450 \mu \mathrm{mol} / \mathrm{min}$, $I=0.66$.
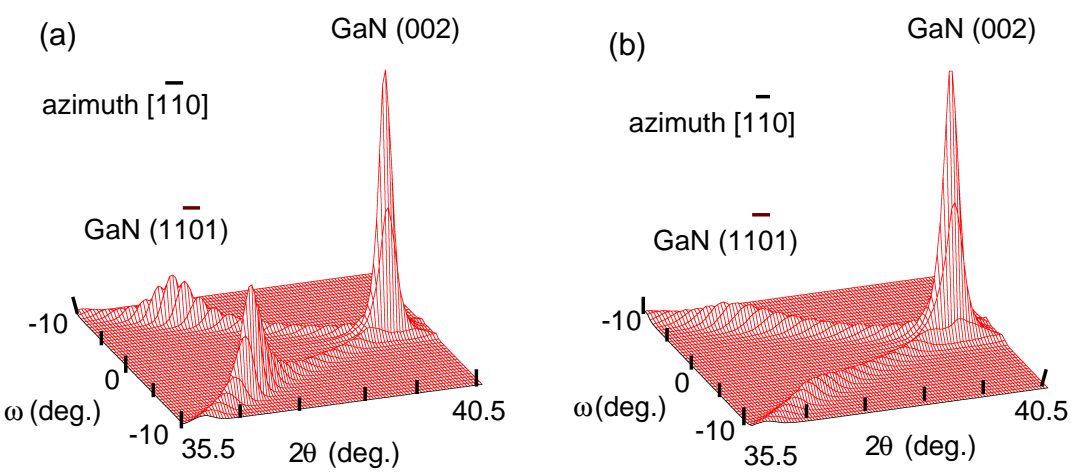

Figure 2. Three-dimensional graphs of reciprocal space maps of (a) c-GaN and (b) cGaNAs $\left(\mathrm{AsH}_{3}\right.$ flow rate was $\left.90 \mu \mathrm{mol} / \mathrm{min}\right)$ with $\mathrm{X}$-ray beams incident along the $<1010>$ azimuth. 


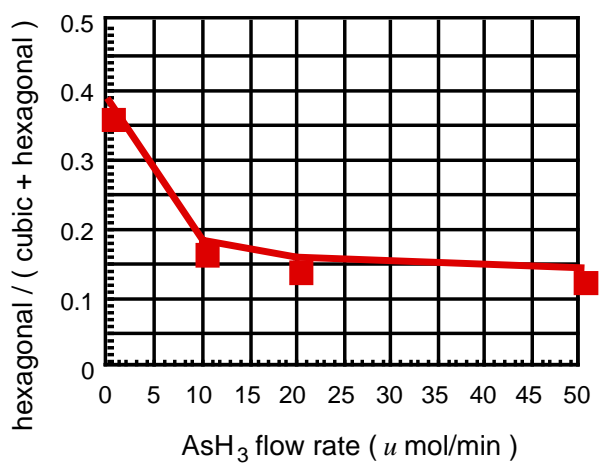

Figure 3. Concentration of the hexagonal phase in the c-GaN layers with various $\mathrm{AsH}_{3}$ flow rates.

diffraction-peak intensity from h-GaN (1-101) on (111) B surfaces was much larger than from h-GaN on (111) A surfaces; this is consistent with that the grains at the c-GaN surface have (111) B facts and do not have (111) A facets. Interestingly, with $\mathrm{AsH}_{3}$ flow, the diffraction intensity from h-GaN (1-101) becomes significantly weaker. This tendency was common to these samples with $\mathrm{AsH}_{3}$ flow under $225 \mu \mathrm{mol} / \mathrm{min}$. We estimated the concentration of the hexagonal phase in the c-GaN layers by comparing with the integrated diffraction intensities of c-GaN (002) and h-GaN (1-101) [10]. It is considered that with $\mathrm{AsH}_{3}$ flow during the c-GaN growth, As adsorbs on the GaN (001) surface and prevents the generation of (111) facets, resulting in maintaining the surface flatness. This smooth surface is favorable to $\mathrm{c}-\mathrm{GaN}$ growth, because $\mathrm{h}-\mathrm{GaN}$ is easy to grow with its c-axis parallel to the <111> directions. Figure 3 shows the concentration of the hexagonal phase in epilayers at different $\mathrm{AsH}_{3}$ flow rates. It was confirmed that with increasing the $\mathrm{AsH}_{3}$ flow the concentration of the hexagonal phase significantly decreased to $15 \%$. That is, this growth condition is very effective for suppressing of hexagonal GaNAs.

Figure 4 shows SIMS depth profiles of the samples with $\mathrm{AsH}_{3}$ flow of 0 and 225 $\mu \mathrm{mol} / \mathrm{min}$. The As intensity of the samples with an $\mathrm{AsH}_{3}$ flow rate of $225 \mu \mathrm{mol} / \mathrm{min}$ became larger, as large as 20 in the same scale. The As counts increased according to the increase in the $\mathrm{AsH}_{3}$ flow rate up to $225 \mu \mathrm{mol} / \mathrm{min}$. Therefore, As was actually incorporated in the c-GaN layers by the $\mathrm{AsH}_{3}$ flow. When the $\mathrm{AsH}_{3}$ flow rate was 450 $\mu \mathrm{mol} / \mathrm{min}$, GaNAs polycrystals were observed in the GaNAs layer. In order to further incorporate the As into GaNAs, the growth condition must to be further improved. 

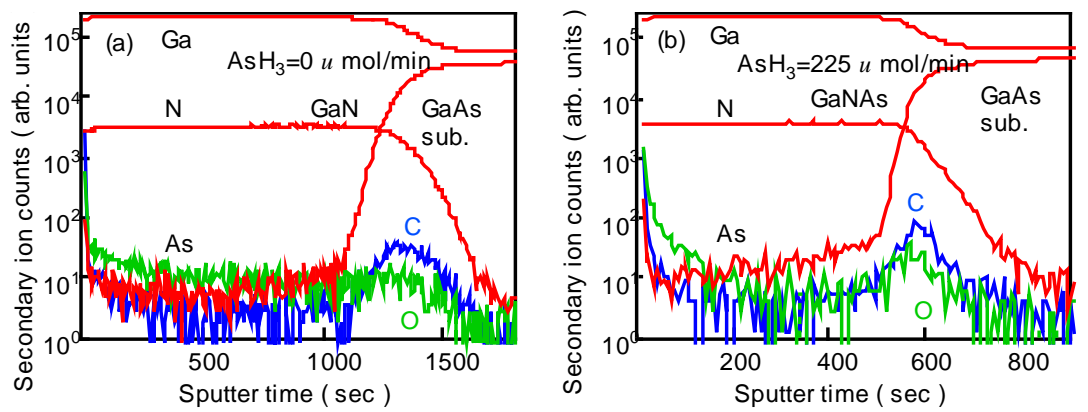

Figure 4. SIMS depth profiles of (a) c-GaN (without $\mathrm{AsH}_{3}$ flow), (b) c-GaNAs $\left(\mathrm{AsH}_{3}\right.$ flow rate was $225 \mu \mathrm{mol} / \mathrm{min}$ ).

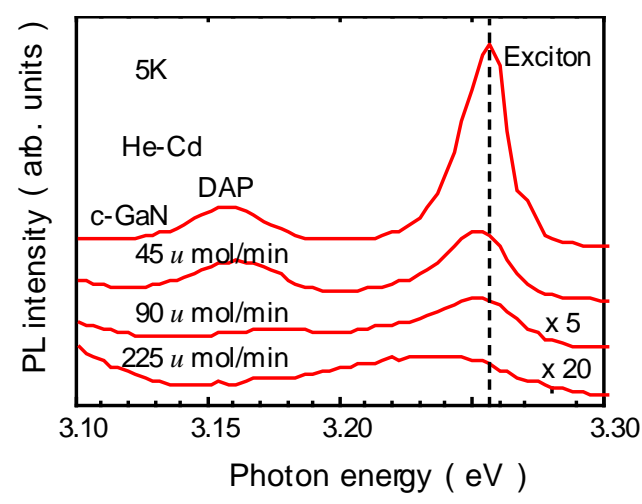

Figure 5. PL spectra of near-band edges with various $\mathrm{AsH}_{3}$ flow rates at 5k.

We next carried out a photoluminescence (PL) measurement of GaNAs grown at different $\mathrm{AsH}_{3}$ flow rates to investigate the optical properties. The two main peaks were observed. That is, the excitonic emission at $3.256 \mathrm{eV}$ and the donor-acceptor pair recombination at $3.156 \mathrm{eV}$ were observed in the PL spectrum of c-GaN. With increasing the $\mathrm{AsH}_{3}$ flow rate, the excitonic emission became weak and the deep luminescence band around $2.8 \mathrm{eV}$ became dominant. Figure 5 shows the near-band-edge emission of the GaNAs PL spectra. With increasing the $\mathrm{AsH}_{3}$ flow rate, the exciton peak shifts toward lower energies and becomes broad. This is due to As incorporation in the $\mathrm{c}-\mathrm{GaN}$ layer. The maximum value of the peak shift is $20 \mathrm{meV}$, which corresponds to approximately $0.1 \%$ As concentration, as estimated from the bowing parameter of hGaNAs (19.6 eV) [5]. 


\section{CONCLUSION}

The hexagonal domain suppression-effects in c-GaNAs grown by MOCVD using DMHy were investigated. GaNAs samples were grown at different $\mathrm{AsH}_{3}$ flow rates $(0$ $450 \mu \mathrm{mol} / \mathrm{min})$ at $1193 \mathrm{~K}$. As a result, three types of surface morphologies were obtained: the first was obtained as a smooth surface $\left(\mathrm{AsH}_{3}=0 \mu \mathrm{mol} / \mathrm{min}\right)$; the second was a smooth surface having small and isotropic grains ( $\left.\mathrm{AsH}_{3}: 45 \sim 225 \mu \mathrm{mol} / \mathrm{min}\right)$; and the third involved three-dimensional surface morphologies (above $450 \mu \mathrm{mol} / \mathrm{min}$ of $\mathrm{AsH}_{3}$ flow rate). Furthermore, it was confirmed using X-ray diffraction that the mixing ratio of hexagonal GaNAs in cubic GaNAs decreased along with an increase in the $\mathrm{AsH}_{3}$ flow rate. We could obtained a GaNAs having a cubic component above $85 \%$ at $\mathrm{AsH}_{3}$ flow rates above $20 \mu \mathrm{mol} / \mathrm{min}$. Therefore, the MOCVD growth method using $\mathrm{AsH}_{3}$ and DMHy was effective for suppressing hexagonal GaNAs.

\section{ACKNOWLEDGMENTS}

This work was supported by NEDO/JRCM's "The Light for the 21' Century" Japanese national project.

\section{REFERENCES}

1. L. Bellaiche, S. - H. Wei and A. Zunger, Appl. Phys. 70, 3558 (1997).

2. S. - H. Wei and A. Zunger, Phys. Rev. 76, 664 (1996).

3. G. B. Stringfellow, J. Electrochem. Soc. 119, 1780 (1972).

4. G. B. Stringfellow, J. Cryst. Growth. 27, 21 (1974).

5. K. Iwata, H. Asahi, K. Asami, R. Kuroiwa and S. Gonda, Jpn. Appl. 37, 1436 (1998).

6. K. Iwata, H. Asahi, K. Asami and S. Gonda, Jpn. Appl. Phys. 35, L1634 (1996).

7. H. Tampo, H. Asahi, K. Iwata, M. Hiroki, K. Asami and S. Gonda, Technical Report of Jpn. IEICE. 98, ED98 (1998).

8. J. Wu, M. Kudo, A. Nagayama, H. Yaguchi, K. Onabe and Y. Shiraki, to be published in Phys. Stat. Sol. (b).

9. T. S. Cheng, L. C. Jenkins, S. E. Hooper, C. T. Foxon, J. W. Orton and D. E. Lacklison, Appl. Phys. Lett. 66, 1509 (1995).

10. H. Tsutchiya, K. Sunaba, S. Yonemura, T. Suemasu, and F. Hasegawa, Jpn. J. Appl. Phys. 36, L1 (1997). 